\title{
LE CONOSCENZE IN FISICA ALL'INIZIO DEI CORSI UNIVERSITARI IN ITALIA
}

\author{
IANNIELLO,M.G. ${ }^{1}$, MAYER, M. ${ }^{2}$, SCALZO, F., STILLI, R. y VICENTINI MISSONI, M. ${ }^{+}$ \\ ${ }^{\mathrm{t}}$ Dip. Fisica, Università «La Sapienza», Roma \\ ${ }^{2}$ Centro Europeo dell Educazione, Frascati, Roma
}

\begin{abstract}
SUMMARY
The article reports the results of an investigation on Italian university students concerning physics knowledge related to common sense schemes at the beginning of the university courses. The results of the diagnosis were communicated both to the students and to the teachers of the courses. The communication enabled the teachers, in the didactical practice, to pinpoint and discuss with the students the discrepancies between the scientific newtonian scheme and common sense scheme. A posttest, given at the end of the course, gives the indication that this practice is effective in inducing a change in the students ideas, notwithstanding the fact that no complete understanding of the scientific viewpoint is reached by the majority of students.
\end{abstract}

\section{INTRODUZIONE}

Uno dei problemi connessi con la ricerca sulle rappresentazioni mentali degli studenti (Driver e Easley 1978, McDermott 1984, Jagger 1985, Vicentini Missoni 1989, AAVV 1989), è quello di indure un cambiamento concettuale verso gif schemi scientifici, a partire dagli schemi di senso comune. Fino ad oggi diversi esperimenti didattici sono stati svolti in questa direzione (Halloun e Hestenes 1985, Gunstone 1987, Pines 1985), senza tuttavia arrivare a risultati definitivi se non per il suggerimento, apparentemente ovvio, che sia gli insegnanti sia gli studenti devono essere consapevoli della discrepanza tra diversi schemi di conoscenza per poter superare le «barriere critiche alla comprensione» (Hawkins 1985). Il suggerimento è ovvio nel senso che da sempre i buoni insegnanti sono consapevoli del fatto che la pratica di insegnamento, per essere efficace, deve partire dagli «errori» degli studenti (vedi per esempio Enriques 1936 , Diesterweg 1835). Dal momento che la ricerca sulle rappresentazioni mentali ha dato un contributo a questa consapevolezza esplicitando alcuni degli schemi di conoscenza di senso comune che sono alla base dei fraintendimenti degli studenti (Jung 1985), riteniamo importante diffondere tali risultati al di fuori delia cerchia ristretta dei ricercatori che se ne sono occupati, a partire in primo luogo dai docenti di fisica dei corsi di base a livello universitario. A tal fine abbiamo somministrato all'inizio dei corsi un «test diagnostico», o pretest, i cui risultati sono stati esplicitamente tenuti in conto dai docenti durante l'insegnamento e successivamente, alla fine del corso, abbiamo proposto un posttest in grado di rilevare l'efficacia del procedimento nel modificare gli schemi di ragionamento degli studenti.

\section{IL TEST DIAGNOSTICO}

Il gruppo che ha condotto l'indagine ha scelto di indirizzare la diagnosi verso quei temi sui quali la ricerca sulle rappresentazioni mentali aveva già fornito domande ben formulate e risultati attendibili.

I temi prescelti hanno pertanto riguardato a) le forze, il movimento e l'equilibrio; b) la pressione; c) la gravità. Per ognuno di questi temi presentiamo di seguito gli schemi di senso comune insieme alle domande che sono state selezionate per l'indagine.

\section{a) Forze, movimento e equilibrio}

Nell'ambito della dinamica gli schemi di senso comune tendono a operare una distinzione tra:

a1) un movimento «naturale» (la caduta lungo la verticale); 
a2) un movimento «violento» (un oggetto lanciato in aria);

a3) un movimento dovuto al trasporto da parte di un altro corpo o di una persona.

Nel caso (a2) la descrizione-spiegazione del fenomeno è accompagnata dall'uso di un concetto che costituisce un misto tra i concetti físici di forza, energia e impulso. Questo concetto, che è stato chiamato «capitale đi forza» (Viennot 1979) o anche "forza di spinta o impulso" (McCloskey 1983), corrisponde ad attribuire al corpo in moto una forza intema, funzione della velocità del corpo e che si consuma durante il moto, il quale termina, quindi, quando il capitale di forza è stato interamente dissipato.

Nel caso (a3), quando un corpo si muove solidaimente a un altro e poi viene lanciato in aria o abbandonato a se stesso, se precedentemente si muoveva di moto traslatorio "dimentica" immediatamente il suo stato di moto (Saltiel 1981), mentre se si muoveva di moto circolare conserva memoria del suo stato di moto e descrive una traiettoria curva (Caramazza 1980).

Per ricavare informazioni sul concetto di forza (nel caso a2) abbiamo scelto le domande riportate in figura 1 . Nel test del calciatore (Mayer 1987), le risposte nelle quali si afferma la presenza, durante il moto, della « forza del calcio» rivelano l'adozione di uno schema di «capitale di forza». Lo stesso si può dire nel test del "gioco a palla» (Watts 1981) per ogni risposta differente dalla A e nel test del "giocoliere" per le risposte che indicano forze diverse (Viennot 1979).

Al fine di verificare la presenza e la coerenza dello schema di senso comune nel test diagnostico abbiamo inserito anche alcune domande più tradizionali (sfera che rimbalza, sfera lasciata cadere nello stesso istante in cui viene lanciato obliquamente un proiettile, grafico accelerazione-tempo di un corpo in caduta, due sfere di peso diverso lanciate verso l'alto).

Per conoscere le idee riguardanti l'inerzia (caso a3) abbiamo scelto le domande riportate in fig. 2. Nel problema della giostra (Mayer 1987) le traiettorie curve mostrano la presenza di uno schema in cui il corpo conserva memoria del moto circolare, in analogia con lo schema dell'impulso ipotizzato da Caramazza e altri (1980). Nel test del «tapis roulant» (Viennot 1979) la risposta A (a meno che non sia chiamata in causa la resistenza dell'aria) è indice di uno schema in base al quale il corpo trasportato non conserva il moto orizzontale. Viceversa, nel test della «barca», l'avere indicato una differenza di velocità tra le due palline di ping-pong mostra che si è fatta una specie di composizione di moti orizzontali.

Infine, abbiamo usato il test dei due mattoni (fig. 3, Gunstone 1987), per analizzare le idee degli studenti sull'equilibrio. In questo caso gli schemi di senso comune suggeriscono che l'equilibrio, per ragioni di simmetria, si abbia solo quando i blocchi sono alla stessa aliezza.

\section{b) Pressione}

La gravità e la pressione dell'aria sono fortemente correlati all'interno degli schemi di senso comune (Ruggiero et al. 1984, Mayer 1987) che vedono la prima come effetto di una pressione agente in direzione verticale. I test sul concetto di pressione sono riportati in fig. 4 . Nei « tubi a $U$ » le risposte che danno una preferenza ad uno dei tre indicano l'importanza che viene attribuita alla forma. Nella domanda del palloncino nella piscina (Mayer 1987) la scelta di A mostra come la pressione dell'acqua venga vista agire solo sulla verticale, mentre l'ipotesi che il palloncino scoppi, con il crescere della pressione, evidenzia come l' attenzione si focalizzi in genere solo sulla pressione esterna. Infine il test della cannuccia può rilevare un ricorso alla «forza di vacuo».

\section{c) Gravità}

A scuola si studia che la gravità è dovuta alla Terra, che la Terra ha una forma sferica, e che la Luna le ruota intomo. Ricerche sulle idee dei bambini in Israele e negli USA (Nussbaum e Novak 1976) e su quelle di adulti e bambini in Italia (Duprè et al. 1981) mostrano che non c'è una completa coerenza tra queste nozioni scolastiche e le idee relative alla gravità intorno alla Terra e sulla sua superficie.

Per analizzare le idee degli studenti in proposito abbiamo impiegato le domande riportate in fig. 5 .

Per il test della «Luna», ogni risposta per Ia quale la persistenza del moto è dovuta all'assenza di forze, mostra che la gravità è intesa come una forza che agisce solo in una regione definita di spazio intorno alla Terra. Nel test del «tunnel nella Terra» una gravità «assoluta» e rivolta verso il basso è una ragione perché il sasso continui la sua traiettoria rettilinea anche fuori dal foro, mentre un equitibrio al centro della Terra indica come a questo punto sia attribuito un significato particolare. Nel test può essere considerata corretta sia la risposta che prevede infinite oscillazioni, sia quella che prevede oscillazioni smorzate con arresto al centro. Risposte incoerenti per le due formulazioni della domanda (tunnel verticale e orizzontale) possono mostrare quanto sia profondamente radicata I' idea che il peso abbia una direzione «verso il basso».

\section{RISULTATI DELLA RICERCA}

Il test è stato proposto a studenti del primo anno dei corsi di laurea in fisica e matematica della Università «La Sapienzay di Roma, distribuiti in corsi parallelit tenuti da docenti diversi (tab. I). La prova, somministrata all' inizio del corso di fisica, era anonima; tuttavia è stato chiesto agli studenti di identificare con una sigla (le prime cinque cifre del numero telefonico) la propria copia in modo che fosse possibile rintracciarla sia per discussioni dichiarimento sia per poter operare un confronto individuale pre-post test. 


\section{NOTE}

1 In htalia sono stati anche raccolti dati in altre sedi universitarie (Cosenza, Messina) su studenti di fisica e di ingegneria i cui risultati sono in sostanziale accordo con quelli qui presentati.

2 Gli studenti nelle Università italiane seguono corsi di lezione in gruppi di 150 e sono suddivisi in sottogruppi di circa 30 per

\section{RIFERIMENTI BILIOGRAFICI}

AAVV, 1989. Students' Conceptions in Science, International Journal of Science Education, Special. Issue, vol. II

BATTIMELLI, G., DUPRE, F., IANNIELLO, M.G., STILLI, R., VICENTINI MISSONI, M,, 1990. Conoscenza della Fisica all'ingresso e alla fine dei corsi universitari di base, La Fisica nella Scuola, 23, pp. 104-115.

CARAMAZZA, A., MCCLOSKEY, M., GREEN, B., 1980. Curvilinear motion in the absence of external force: naive beliefs about the motion of objects, Science, 210, pp. 11391141 .

CARRASCOSA, J., GIL-PÉREZ, D., 1990. What to do about Science «Misconceptions», Science Education, 74, 59, pp. $531-540$

CLEMENT, J., 1982. Students' preconceptions in introductory mechanics, American Journal of Physics, 50, pp. 66-71.

DIESTERWEG, F.A.M., 1835. Wegweiser zur Bildung für deutsche Lehrer, in Heilmann, P., Quellenbuch der Pädagogik (Durrsche Buchhandlung: Leipzig 1909).

DRIVER, R., EASLEY, J., 1978. Pupils and paradigms: a review of the literature related to concept development in adolescent science student, Studies in Science Education, 5, pp. 61-84.

DUPRE', F., NOCE, G., VICENTINI MISSONI, M., 1981. Modelli fisici prenewtoniani nelle conoscenze degli adulti, Scuola e Città, 2, pp. 53-64, 1984. Die Gestalt der Erde und die Schwerkraft: Common Sense Wissen von Erwachsenen, Physica Didactica, 11; pp. 3-22.

ENRIQUES, F., 1936. Il significato della storia del pensiero scientifico, en G. Giorello (a cura di), L'immagine della scienza, Il Saggiatore, Milano 1977

GUNSTONE, R.F., 1987. Student understanding in mechanics: a large population survey, American Journal of Physics, 55, pp. $691-696$.

HALLOUN, I. A., HESTENES, D., 1985. Common sense concepts about motion, American Journal of Physics, 53,pp. $1056-1065$.

HAWKINS, D, 1985. Critical barriers in understanding science. Internal Report Boulder, Colorado. seguire attività di risoluzione a problemi condotte da docenti diversi dai titolari dei corsi.

3 Nelia Università di Roma gli studenti non sono obbligati a seguire le lezioni ma possono decidere il proprio metodo di studio. Non necessariamente gli studenti che frequentano it corso sono i migliori.

HEWSON, M.G., HEWSON, P.W.,1984. Effects of instruction using students' prior knowledge and conceptual change strategies on science learning, European Journal of Science Education, 6, pp. 1-6.

JAGGER, J. M., 1985. A review of the research into the learning of mechanics, in Studies in mechanics learning. Center for Studies in Science and Math Education, University of Leeds, pp. 87-113.

JUNG, W., 1985. Schulervorstellungen in Physikunterricht ein didaktisches Programm, Physica Didoctica, 12, pp. 11-22.

MAYER, M., 1987. Conoscenza scientifica e conostenza di senso comune, $\mathrm{Ph}$. D. Thesis, Roma, Italy.

MCDERMOTT, L. C., 1984. Research on conceptual understanding in mechanics, Physics Today, July 1984, pp. 24-32.

NUSSBAUM, J., NOVAK, J. D., 1976. An assessment of children's concepts of earth utilizing structured interviews, Science Education, 60, pp. 535-550.

PINES, L.A., NOVAK, J.D., 1985. The interaction of audio. tutoriat instruction with student prior knowledge: a proposed qualitative, case study methodology, Science Education, 69 , pp. 213-220.

RUGGIERO, S., CARTELLI, A., DUPRE', F., VICENTINI MISSONI M., 1985. Weight, gravity and airpressure, European Journal of Science Education, 7, pp. 181-194.

SALTIEL, E., 1981. Kinematic concepts and natural reasoning: study of comprehension of galiean frames by science students European Journal of Science Education, pp. 110-111.

VICENTINI MISSONI, M., 1989. Etat des recherches sur les schémas, de connaissance communes, alternatifs à la connaissance scientifique, Psychologie génétique et didactique des sciences, pp. 127-156 (Ed. Giordan, Lang: Berna).

VIENNOT, L., 1979. Le raisonnement spontané en dynamique élémentaire. (Hermann: Paris).

WATTS, D. M., ZYLBERSZTAJN, A., 1981. A survey of some children's ideas about force, Physics Education, 16, pp. 360- 365 . 


\section{PRETEST}

Test - Fig. 1

CALCIATORE. Quando il pallone è in aria su di esso agiscono: A. Nessuna forza. B. La forza di gravità. C. La forza del calcio. D. La forza di attrito.

G1OCO A PALLA. Sceglì la figura che rappresenta meglio la forza agente sulia palla mentre è in aria.

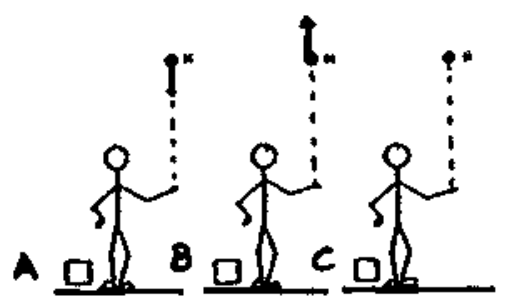

GIOCOLIERE. Dire se le forze agenti sulle sei palle quando si trovano alla stessa altezza e trascurando la resistenza dell'aria, sono uguali o diverse.

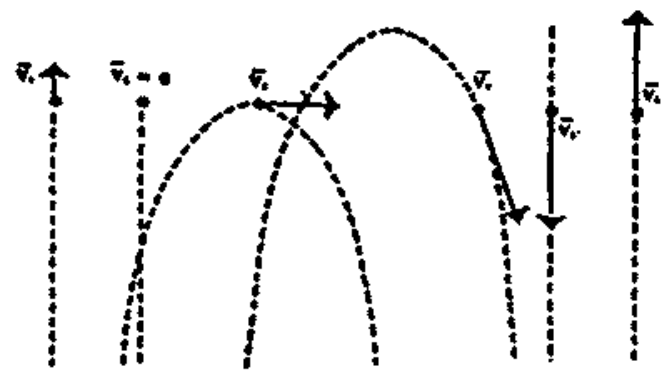

Test-Fig. 2

GIOSTRA. Traccia la traiettoria di una palla lanciata da un punto di una giostra che sta ruotando, affinché la palla raggiunga un bersaglio posto in $\mathbf{P}$.

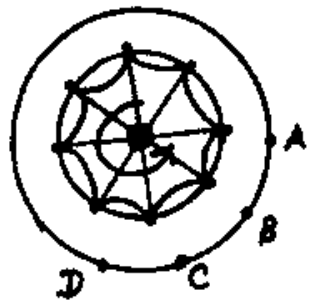

TAPIS ROULANT. Un ragazzo sta lanciando in aria una palla in verticale mentre sta su un tapis roulant. La palla cadra: $A$. Dietro al ragazzo.; B. Nelle sue mani; C. Davanti al ragazzo; D. Altro.

BARCA. Su una barca in moto a velocità costante in un mare calmo, due viaggiatori stanno giocando sotto coperta a ping pong su un tavolo orientato in direzione da prua a poppa. La velocità della palla sarà maggiore: $A$. Se viene lanciata dal giocatore che guarda a poppa; $B$. Se viene lanciata dal giocatore che guarda a prua; $C$. Non c'è differenza di velocità; D. I dati non sono sufficienti.
Test - Fig. 3

MATTONI. Due mattoni identici collegati da una fune vengono lasciati a diverse altezze. A. Rimarranno nella stessa posizione; B. Si muovono verso la posizione corrispondente alla stessa altezza; C. Oscillano intomo alla posizione B; D. ll mattone più basso raggiunge il pavimento.

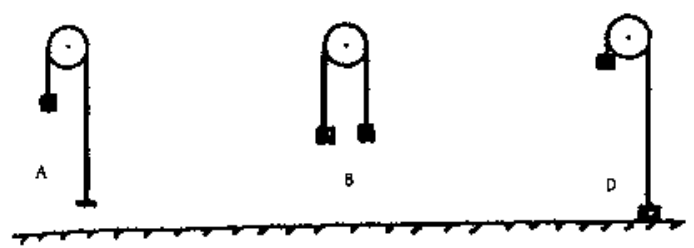

Test-Fig. 4

TUBI A U. Per misurare una differenza di pressione può essere usato uno qualunque dei tubi in figura. Per quale di questi la scala sarà lineare?

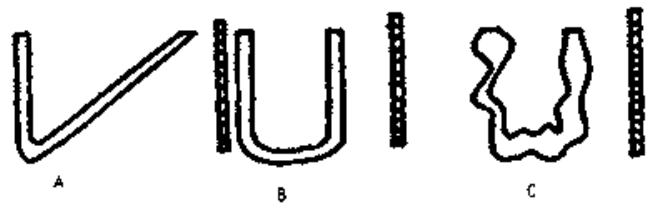

PALLONCINO NELLA PISCINA. Un palloncino pieno d'aria viene fissato al fondo di una piscina profonda 4 metri. Quale delle figure rappresenta meglio la forma del pallone quando la piscina è piena d'acqua?

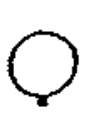

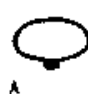

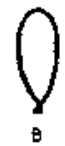

$\theta$

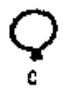

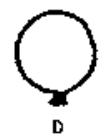

CANNUCCIA. Si pud bere un liquido con una cannuccia perché: A. La forza del vuoto tira su il liquido; B. C'è una differenza di pressione quando si aspira; $\mathrm{C}$. Per capillarità.

Test- Fig. 5

TEST DELLA LUNA. La Luna non cade sulla Terra perché: A. Su di essa non agiscono forze; B. Esistono forze che le proibiscono di cadere:..... C. La forza che la Luna esercita suila Terra è uguale e opposta alla forza che la Terra esercita sulla Luna; D. La Luna cade però la sua velocità la mantiene in moto nell'orbita.

TUNNELL NELLA TERRA. 1. Se si lancia un sasso in un buco scavato attraverso la Terra per il centro, come in figura a, il sasso: A. Esce e si perde nello spazio; B. Si ferma al centro della Terra; C. Oscilla tra i due estremi. 2 . Traccia in fig. b la traiettoria seguita da una palla lasciata ad una estremitả del buco.
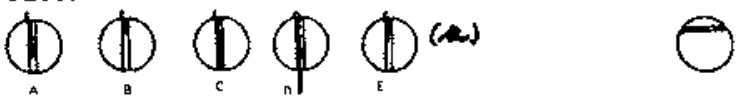


\section{Posttest - Fig. 6}

CANNONE. Disegnare in tre punti della traiettoria descritta da tna palla di cannone la direzione della forza agente sul proiettile.

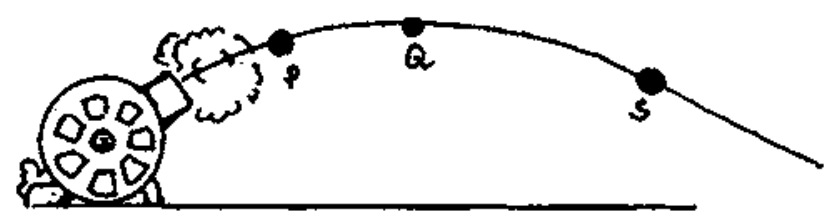

RAZZO. Un razzo si muove nello spazio da $P$ a $Q$. In $Q$ si accendono i motori e il razzo si sposta in $R$ dove si spengono i motori. Scegliere tra le figure quella che rappresenta meglio la traiettoria seguita dal razzo.

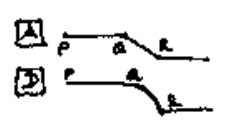

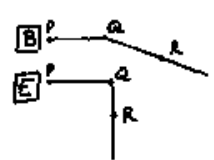

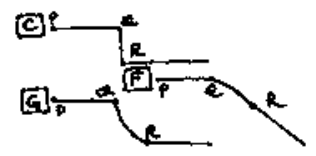

CORRIDORE. Un corridore che si muove a velocità costante lascia cadere una sfera di piombo quando è in A. Dove toccherà terra la sfera?

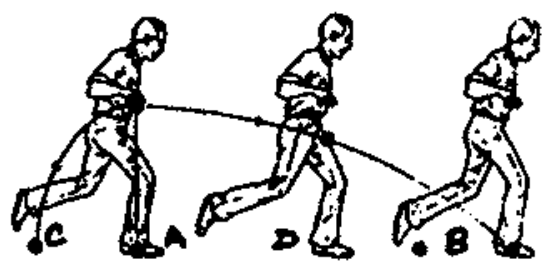

SPECCHIO. Uno specchio ha spessore $1 \mathrm{~cm}$ e superficie di 1 $\mathrm{m}^{2}$. La pressione dell'aria sulla faccia riflettente sara minima quando: A. Tale faccia è orizzontale e rivolta verso l'alto; $\mathbf{B}$. Tale faccia è orizzontale e rivolta verso il basso; C. Lo specchio è disposto verticalmente; D. Non si ha minimo di pressione. 